\title{
NONLINEAR DIFFERENCE EQUATIONS CONTAINING A PARAMETER
}

\author{
C. F. STEPHENS
}

1. Formulation of the problem. Consider the system of nonlinear difference equations having the form

$$
\begin{aligned}
y_{i}(x+1)=x^{-1} \sum_{i=1}^{n} b_{i j}(x) y_{j}(x)+f_{i}\left(y_{1}(x), \cdots, y_{n}(x) ; p ; x\right), \\
f_{i}(0, \cdots, 0 ; 0 ; x) \equiv 0 \quad(i=1, \cdots, n),
\end{aligned}
$$

where the $f_{i}$ are analytic functions of $y_{i}(x)$ and the constant parameter $p$, continuous functions of the complex variable $x$, and bounded for all values of the $y_{i}(x), p$, and $x$ for which

G:

$$
\left|y_{i}(x)\right| \leqq r_{i} ; \quad|p| \leqq \rho ; \quad|x| \geqq K .
$$

The $f_{i}$ contain terms in $p$ alone and can be developed into convergent power series in the $y_{i}(x)$ and $p$. The $b_{i j}(x)$ are any continuous and bounded functions of $x$ which are defined in the domain $G$. We could have taken the parameter $p$ to be a periodic function of period 1 .

In other words, we may write

$$
f_{i}=g_{i}(x, p)+h_{i}\left(y_{1}(x), \cdots, y_{n}(x) ; p ; x\right),
$$

where

$$
\begin{aligned}
g_{i}(x, p) & =x^{-1} A_{i 1}(x) p+\sum_{j=2}^{\infty} A_{i j}(x) p^{i}, \\
h_{i} & =\sum_{m=2}^{\infty} \Sigma a_{\alpha_{0}, \alpha_{1}}^{(i)} \ldots, \alpha_{n}(x) p^{\alpha_{0}} y_{1}^{\alpha_{1}}(x) \cdots y_{n}^{\alpha_{n}}(x),
\end{aligned}
$$

and

$$
\Sigma=\left(\alpha_{0} \neq m ; \alpha_{0}, \cdots, \alpha_{n} \geqq 0 ; \alpha_{0}+\cdots+\alpha_{n}=m\right) .
$$

The $A_{i j}(x)(j=1,2, \cdots)$ and $a_{\alpha_{0}, \alpha_{1}, \ldots, \alpha_{n}}^{(i)}(x)$ are any continuous and bounded functions of $x$ such that the right members of (2a) and (2b) converge, where the functions $A_{i 1}(x)$ can conceivably be identically zero.

In a recent work ${ }^{1}$ the author made certain investigations concern-

Presented to the Society, April 30, 1949; received by the editors February 3, 1949.

1 C. F. Stephens, Nonlinear difference equations analytic in a parameter, Trans. Amer. Math. Soc. vol. 64 (1948) pp. 268-282. 
ing systems of nonlinear difference equations similar to system (1). However, the functions in the right members of these systems corresponding to the $f_{i}$ are more restrictive as functions of $x$. A system similar to (1) in which the functions corresponding to the $b_{i j}(x)$ are more restrictive as functions of $x$ was first considered by the author in his doctoral dissertation, which was prepared at the University of Michigan under the supervision of J. A. Nyswander. Also, the results contained in the first two chapters of the author's paper to which reference is made, with slight changes, were obtained in his doctoral thesis, Theorem 6 being a generalization of a theorem considered there. The procedure for investigating solutions of system (1) which will be given in this paper is different from and simpler than the one considered in the author's dissertation.

2. Preliminary results. The proof of the existence of a solution of system (1) which is analytic in $p$ and continuous in $x$ is based on the following result. Let us consider the system of nonlinear difference equations

$$
\begin{gathered}
z_{i}(x+1)=l_{i}\left(z_{1}(x), \cdots, z_{n}(x) ; p ; x\right), \\
l_{i}(0, \cdots, 0 ; p ; x)=a_{i}(x, p) \not \equiv 0 \quad(i=1, \cdots, n),
\end{gathered}
$$

where the $l_{i}$ are analytic functions of the $z_{i}(x)$, continuous functions of $x$ and $p$, and can be developed into convergent power series starting with terms independent of $z_{i}(x)$.

Let $C$ be a circle with center at the origin and radius $R^{\prime}(=R+1)$. Draw tangents $t$ and $t^{\prime}$ to the circle $C$ at the points where the circle cuts the axis of imaginaries. Denote by $D$ all points not interior to the domain bounded by that part of the circle $C$ which lies to the left of the axis of imaginaries and the parts of tangents $t$ and $t^{\prime}$ which do not lie to the left of this axis. Thus the domain $D$ contains no part of the positive axis of reals.

The following lemma is similar to a result obtained previously by the author, ${ }^{2}$ and can be proved by similar methods:

Lemma. If we can determine positive constants $\lambda, R$, and $r$ such that

$$
\left|l_{i}\left(z_{1}(x), \cdots, z_{n}(x) ; p ; x\right)\right|<r / 4 n
$$

for

$$
\left|z_{i}(x)\right| \leqq r, \quad|p| \leqq \lambda, \quad|x| \geqq R,
$$

then the system of equations (3) has a solution having the form

2 Ibid. \$9. 


$$
z_{i}(x)=\sum_{k=1}^{\infty} C_{i}^{(k)}(x, p) \quad(i=1, \cdots, n)
$$

where the right members of equations (4) are absolutely and uniformly convergent in $x$ and $p$ for $x$ in the domain $D$ and for $|p| \leqq \lambda$.

For the proof, a new system of equations

$$
z_{i}(x+1)=t l_{i}\left(z_{1}(x), \cdots, z_{n}(x) ; p ; x\right) \quad(i=1, \cdots, n)
$$

is introduced, containg a new parameter $t$. A solution

$$
z_{i}(x)=\sum_{k=1}^{\infty} C_{i}^{(k)}(x, p) t^{k}
$$

of this system is computed by substitution into (5) and comparison of like powers of $t$. By a method of dominant analytic implicit equations the series is shown to converge for $t=1$, and when $t$ is set equal to 1 a solution of (3) is obtained in the form (4).

3. Proof. We shall now prove the existence of a solution of system (1) which is analytic in the parameter $p$ and continuous in $x$. In order to accomplish this aim, we shall transform the system of equations (1) into a system such that the conditions of our lemma are satisfied. Let us make the transformation

$$
y_{i}(x)=p z_{i}(x) \quad(i=1, \cdots, n) .
$$

Then the system of equations (1) is transformed into the system

$$
\begin{aligned}
z_{i}(x+1)= & x^{-1} \sum_{j=1}^{n} b_{i j}(x) z_{j}(x)+x^{-1} A_{i 1}(x)+p \sum_{j=2}^{\infty} A_{i j}(x) p^{j-2} \\
& +p \sum_{m=2}^{\infty} \Sigma a_{\alpha_{0}, \cdots, \alpha_{n}}^{(i)}(x) p^{m-2} z_{1}^{\alpha_{1}}(x) \cdots z_{n}^{\alpha_{n}}(x) \\
\equiv & l_{i}\left(z_{1}(x), \cdots, z_{n}(x) ; p ; x\right) \quad(i=1, \cdots, n) .
\end{aligned}
$$

Let $r$ be the smallest of the numbers $r_{i}$ and let $\mu$ be the smaller of the numbers $\rho$ and 1 . Then we can find positive constants $B, E$ and $H$ such that

$$
\begin{gathered}
\left|\sum_{j=1}^{n} b_{i j}(x) z_{j}(x)+A_{i 1}(x)\right| \leqq B,\left|\sum_{j=2}^{\infty} A_{i j}(x) p^{j-2}\right| \leqq E, \\
\left|\sum_{m=2}^{\infty} \Sigma a_{\alpha_{0}, \alpha_{1}}^{(i)} \cdots, \alpha_{n}(x) p^{m-2} z_{1}^{\alpha_{1}}(x) \cdots z_{n}^{\alpha_{n}}(x)\right| \leqq H,
\end{gathered}
$$

for $\left|z_{i}(x)\right| \leqq r,|p| \leqq \mu,|x| \geqq K$. 
We shall take $\lambda$ to be the smaller of the numbers $\mu$ and $(1 / 3(E+H))$ $\cdot(r / 4 n)$. Also, take $R$ to be the larger of $K$ and $2 B(4 n / r)$. Then

$$
\begin{aligned}
\left|l_{i}\left(z_{1}(x), \cdots, z_{n}(x) ; p ; x\right)\right| & \leqq|p|(E+H)+(1 /|x|) B \\
& \leqq(1 / 3)(r / 4 n)+(1 / 2)(r / 4 n)<r / 4 n,
\end{aligned}
$$

for $\left|z_{i}(x)\right| \leqq r,|p| \leqq \lambda,|x| \geqq R$.

It follows that the system of equations (7) has a solution of the form (4). Also, we know from the properties of the $l_{i}$ and the method of computing the $C_{t}^{(k)}(x, p)$ that each term in the right members of equations (4) is analytic in $p$ and continuous in $x$. Therefore, it follows that these solution-functions of equations (7) are analytic in $p$ and continuous in $x$, since the right members of equations (4) converge absolutely and uniformly in $x$ and $p$. Finally, we obtain from these results and equations (6) that the solution-functions thus obtained for equations (1) are analytic in $p$, continuous in $x$, and vanish identically for $p$ equal to zero.

4. Example $(n=1)$. Consider the equation having the form

$$
y(x+1)=x^{-1} b(x) y(x)+f(y(x) ; p ; x), f(0 ; 0 ; x) \equiv 0,
$$

where

$$
\begin{aligned}
f & =g(x, p)+h(y(x) ; p ; x), \\
g(x, p) & =\sum_{j=2}^{\infty} p^{j}, \\
h(y(x) ; p ; x) & =\sum_{m=2}^{\infty} y^{m}(x) .
\end{aligned}
$$

The power series (9a) and (9b) converge and the function $b(x)$ is bounded and continuous for all values of $y(x), p$, and $x$ for which

$G_{1}: \quad|y(x)| \leqq r<1 ; \quad|p| \leqq \rho<1 ; \quad|x| \geqq K$.

Let us make the transformation

$$
y(x)=p z(x) .
$$

Then (8) is transformed into the equation

$$
\begin{aligned}
z(x+1) & =x^{-1} b(x) z(x)+p \sum_{j=2}^{\infty} p^{j-2}+p \sum_{m=2}^{\infty} p^{m-2} z^{m}(x) \\
& \equiv l(z(x) ; p ; x) .
\end{aligned}
$$


The series $\sum_{m=2}^{\infty} z^{m}(x)$ converges for $|z(x)| \leqq r<1$ and dominates the series $\sum_{m=2}^{\infty} p^{m-2} z^{m}(x)$, since $|p|<1$. Also, the geometric series $\sum_{j=2}^{\infty} p^{j-2}$ converges for $|p|<1$. Therefore, we can find positive constants $B, E$, and $H$ such that

$$
\begin{aligned}
|b(x) z(x)| & \leqq B,\left|\sum_{j=2}^{\infty} p^{j-2}\right| \leqq E, \\
\left|\sum_{m=2}^{\infty} p^{m-2} z^{m}(x)\right| & \leqq B,
\end{aligned}
$$

for

$$
|z(x)| \leqq r ; \quad|p| \leqq \rho ; \quad|x| \geqq K .
$$

Take $\lambda$ to be the smaller of the numbers $\rho$ and $(1 / 3(E+H))(r / 4)$. Also, take $R$ to be the larger of the numbers $K$ and $2 B(4 / r)$. Then

$$
\begin{aligned}
|l(z(x) ; p ; x)| & \leqq(1 /|x|) B+|p|(E+H) \\
& \leqq(r / 2 B(4)) B+(1 / 3(E+B))(r / 4)(E+B)<r / 4,
\end{aligned}
$$

for

$$
|z(x)| \leqq r ; \quad|p| \leqq \lambda ; \quad|x| \geqq R .
$$

We have

$$
\begin{aligned}
& C^{(1)}(x, p)=\sum_{j=2}^{\infty} p^{j-1}=p+p^{2}+p^{3}+\cdots, \\
& C^{(2)}(x, p)=\frac{b(x-1)}{(x-1)}\left(p+p^{2}+p^{3}+\cdots\right) \\
& C^{(3)}(x, p)=\frac{b(x-1) b(x-2)}{(x-1)(x-2)}\left(p+p^{2}+\cdots\right)+p\left(p+p^{2}+\cdots\right)^{2},
\end{aligned}
$$

Therefore, we can write

$$
z(x)=g_{1}(x) p+g_{2}(x) p^{2}+\cdots,
$$

where

$$
\begin{aligned}
g_{1}(x)=1 & +\frac{b(x-1)}{x-1}+\frac{b(x-1) b(x-2)}{(x-1)(x-2)}+\cdots \\
& +\frac{b(x-1) \cdots b(x-n+1)}{(x-1) \cdots(x-n+1)}+\cdots
\end{aligned}
$$

Finally, we have 


$$
y(x)=g_{1}(x) p^{2}+g_{2}(x) p^{3}+\cdots .
$$

Direct substitution of this value for $y(x)$ in (8) shows that $g_{1}(x)$ must satisfy the nonhomogeneous linear difference equation

$$
g_{1}(x+1)=1+\frac{b(x)}{x} g_{1}(x) .
$$

We have

$$
\begin{aligned}
g_{1}(x+1)=1 & +\frac{b(x)}{x}+\frac{b(x)}{x} \frac{b(x-1)}{(x-1)}+\cdots \\
& +\frac{b(x)}{x} \frac{b(x-1) \cdots b(x-n+2)}{(x-1) \cdots(x-n+2)}+\cdots \\
=1 & +\frac{b(x)}{x}\left(1+\frac{b(x-1)}{(x-1)}+\cdots\right. \\
& \left.+\frac{b(x-1) \cdots b(x-n+1)}{(x-1) \cdots(x-n+1)}+\cdots\right) \\
= & 1+\frac{b(x)}{x} g_{1}(x) .
\end{aligned}
$$

Morgan State College 